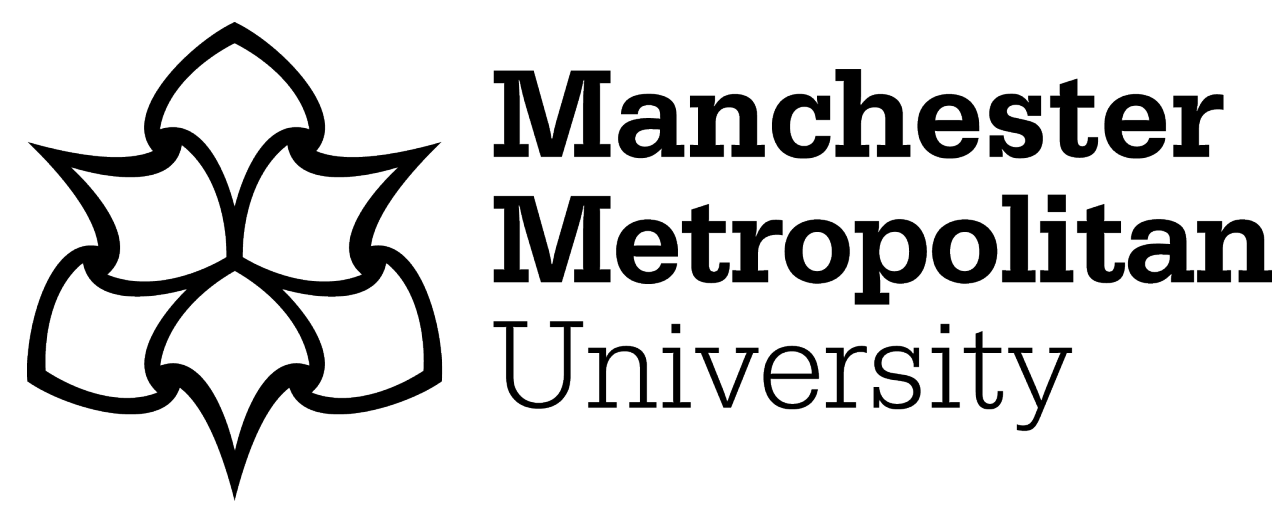

Thomas, C, Dos'santos, T, Cuthbert, M, Fields, C and Jones, PA (2019) The effect of limb preference on braking strategy and knee joint mechanics during pivoting in female soccer players. Science and Medicine in Football, 4 (1). pp. 30-36. ISSN 2473-3938

Downloaded from: https://e-space.mmu.ac.uk/626028/

Version: Accepted Version

Publisher: Taylor \& Francis

DOI: https://doi.org/10.1080/24733938.2019.1667020

Please cite the published version 


\section{The effect of limb preference on braking strategy and knee joint mechanics during pivoting in female soccer players}

Christopher Thomas, Thomas Dos'santos, Matthew Cuthbert, Cara Fields \& Paul Anthony Jones

To cite this article: Christopher Thomas, Thomas Dos'santos, Matthew Cuthbert, Cara Fields \& Paul Anthony Jones (2019): The effect of limb preference on braking strategy and knee joint mechanics during pivoting in female soccer players, Science and Medicine in Football, DOI: 10.1080/24733938.2019.1667020

To link to this article: https://doi.org/10.1080/24733938.2019.1667020

Accepted author version posted online: 11

Sep 2019.

Published online: 17 Sep 2019. 


\title{
The effect of limb preference on braking strategy and knee joint mechanics during pivoting in female soccer players
}

\author{
Christopher Thomas, Thomas Dos'santos, Matthew Cuthbert, Cara Fields and Paul Anthony Jones
}

Directorate of Sport, Exercise and Physiotherapy, University of Salford, Salford, UK

\begin{abstract}
Purpose: The purpose of this study was to explore whether limb preference influences braking strategy and knee joint mechanics during a $180^{\circ}$ pivot task in female soccer players.

Methods: Three-dimensional motion analyses of pivoting on the preferred and non-preferred kicking limbs were performed using 10 Qualisys 'Oqus 7' infrared cameras (240 Hz). Ground reaction forces (GRF) were collected from two AMTI force platforms $(1200 \mathrm{~Hz})$ embedded into the running track to examine penultimate (PEN) and final (FC) contact.

Results: Both preferred and non-preferred limbs involved greater hip (ES $=2.85-3.81)$ and knee joint flexion angles (ES $=5.74-5.78)$ and peak vertical GRFs (ES $=0.87-1.61)$, but lower average vertical (ES $=2.55-3.01)$ and horizontal GRFs (ES = 3.05-3.67) during the PEN compared to the FC. Knee abduction angles were very likely greater $(E S=0.61)$ when turning off the non-preferred limb compared to the preferred limb.

Conclusion: These findings may help us question the role of limb preference during pivoting, yet knee abduction angles and moments should be monitored with caution in female soccer players. Thus, it remains inconclusive the role limb preference plays in change of direction biomechanics for performance and risk of injury.
\end{abstract}

KEYWORDS Deceleration; knee abduction moment; change of direction ability; penultimate contact

\section{Introduction}

Change of direction (COD) is a dominant feature of field- and court-based sport athletes and is defined as the ability to decelerate, reverse or change movement direction and accelerate again, and is considered pre-planned' (Jones et al. 2009). Time-motion analysis data of soccer show players perform the equivalent of $727 \pm 203$ turns during play (Bloomfield et al. 2007), with players performing $45-49$ turns of $90-180^{\circ}$ across all positions. Furthermore, elite soccer athletes perform up to $32 \%$ of directional changes of $180^{\circ}$ (Robinson et al. 2011; Ade et al. 2016). In addition, previous authors suggest a maximaleffort $180^{\circ}$ pivot manoeuvre (i.e. a reversal of direction) is considered to be representative of the nature of competitive soccer match-play (Bloomfield et al. 2007; Greig 2009). Thus, the ability to change direction is important and may strongly influence periods of play whereby athletes are making a break from an opponent, or applying pressure to achieve a turnover (Young et al. 2015). Yet, limb preference has been suggested to play a sex-based role in non-contact anterior cruciate ligament injury, specifically in soccer players (Brophy et al. 2010). Indeed, $74 \%$ (20/27 cases) of males sustained a greater number of non-contact anterior cruciate ligament $(\mathrm{ACL})$ injuries to the dominant limb, compared to $32 \%(10 / 31)$ in females. Thus, female soccer players were more likely to injure their $A C L$ in the non-dominant limb (support/stance) limb, whereas males demonstrated the opposite. Moreover, lower-limb joint postures adopted during COD such as lateral trunk flexion
(Dempsey et al. 2007), knee valgus (Dempsey et al. 2009), limited knee flexion (Kristianslund et al. 2014), lateral foot plant (Jones et al. 2015), and high ground reaction forces (Kristianslund et al. 2014) may invoke high joint loads, may be necessary for performance. However, despite the increasing research into COD ability from a performance perspective, our understanding of limb preference and its influence on lowerlimb mechanics during COD remains a major challenge.

Previous research in female soccer players reported subtle differences in knee mechanics during weight acceptance of $45^{\circ}$ cuts between preferred (kicking) and non-preferred limbs (Brown et al. 2014). However, these authors only focused on the turning limb (final contact $[F C]$ ) and did not consider the role of penultimate contact (PEN) in the braking strategy. Preliminary work suggests that limb preference does not influence COD ability, knee injury risk factors or braking strategy of a $180^{\circ}$ pivot task (Brown et al. 2016). However, the study used a heterogeneous sample of team-sport athletes and a low sample size $(n=8)$. Recent evidence by Jones et al. (2017) found that the PEN plays a role in braking during $180^{\circ}$ pivoting in female soccer players, however participants performed all trials turning off the same limb. Thus, there is inconclusive evidence as to whether limb preference influences the biomechanical characteristics of COD. Moreover, the role of the PEN may vary whether turning off the preferred or non-preferred limb. Thus, further investigation into this topic is warranted. Collectively, though preliminary, these findings could potentially have large practical implications regarding technique 
modification when prescribing COD training for performance and reducing the potential for risk of injury.

Apart from a few (Graham-Smith et al. 2009; Jones et al. 2016a, 2017; Dos' Santos et al. 2017) very little is known about the importance of the PEN during COD, and how body postures and load distribution affect lower-limb kinetics and kinematics during sharper COD. Therefore, COD may be further defined as the events that occur just prior (entry), at 'plant' (occurring between entry and exit) and just following (exit). A recent review (Dos'Santos et al. 2019) suggests that adjustments to body positions in the footfall prior to the COD are required to accomplish deceleration, translation of the body, and reacceleration into a new direction. Furthermore, braking demands have been found to be evenly distributed across approach and execution steps during a $45^{\circ}$ cut, whereas greater impulse and posterior GRF was required in the approach step during a $90^{\circ}$ cut (Havens and Sigward 2015a). However, no previous research has examined limb preference regarding braking strategy in $\mathrm{PEN}$ and $\mathrm{FC}$ during $180^{\circ}$ pivoting in female soccer players. The primary aim of this study was to explore kinematic (lower limb joint angles) and kinetic (GRFs and moments) differences between PEN and FC of pivoting in female soccer players. The secondary aim was to investigate whether differences in kinematics and kinetics exist between preferred and non-preferred limbs. Based on previous research (Brophy et al. 2010), it was hypothesized that participants would exhibit greater knee abduction angles when turning off the non-preferred compared to the preferred limb. We also hypothesized that participants would demonstrate a different braking strategy when turning off the non-preferred limb, compared to the preferred limb.

\section{Methods}

\section{Participants}

Fifteen female soccer players (mean $\pm \mathrm{SD}$; age $=20.6 \pm 0.6$ years; height $=165 \pm 7.0 \mathrm{~cm}$; body mass $=56.6 \pm 6.3 \mathrm{~kg}$ ) participated in the study. All players were registered with soccer clubs playing in the second tier of English Women's Soccer. All players met the inclusion criteria: (1) fully active (i.e. 3 sessions per week) in female soccer competition, (2) did not suffer from an ACL injury and (3) did not suffer from any other lower limb injury within the last 6 months before data collection. Written informed consent was attained from all participants and approval for the study was provided by the University's ethical committee. The study was conducted in accordance with the Declaration of Helsinki.

\section{Procedures}

All participants were fitted with identical size appropriate compression tops (Champion Vapor, Champion, WinstonSalem, NC, USA) and indoor shoes (Balance W490, New Balance, Boston, MA, USA). The leg which a player preferred to kick the ball with was noted as the preferred limb. Testing took place on an indoor synthetic running surface (Mondo, SportsFlex, $10 \mathrm{~mm}$; Mondo America Inc., Mondo, Summit, NJ, USA). All participants performed a modified 505 COD task, turning off the preferred and non-preferred limbs, considered to be representative of the nature of competitive soccer match-play (Greig 2009). In accord with previous research (Brown et al. 2014), the limb which a player prefers to kick the ball with was defined as the preferred limb (right $=11$ participants; left $=4$ participants). All participants performed a standardised progressive warm-up directed by the investigator including various bodyweight lunges and squats, interspersed with footwork and sprint mechanics drills, replicating the athlete's standardised warm-ups before training. This was followed by practice trials of the modified 505 (3 on each limb). The modified 505 involved running towards two force platforms, whereby the first force platform was used to measure GRFs from the PEN foot contact, whilst the 2nd force platform was used to measure GRFs from the FC. Athletes were instructed to sprint to a line marked on the central portion of 2nd force platform, $5 \mathrm{~m}$ from the start, planting their preferred and non-preferred foot on the line, turn $180^{\circ}$ and sprint back $5 \mathrm{~m}$ through the finish. During the test session, all participants performed a minimum of 6 trials on each limb (preferred and non-preferred) in a randomized order and counterbalanced between participants. Total time to complete the tasks was measured using a set of Brower timing lights (Brower Timing Systems, Draper, UT, USA) set at approximate hip height for all participants. Participants were instructed to perform trials with at maximum speed whilst contacting the central portion of the 2nd platform during FC to ensure a homogeneous distance of travel between trials and without prior stuttering or prematurely turning prior to FC. Verbal feedback was provided to rectify any of the abovementioned aspects on subsequent trials. Each subject was allowed time prior to data collection to identify their exact starting point to ensure an appropriate force platform contact. The fastest 3 trials were used for further analysis.

The procedures have been reported previously (Jones et al. 2016a), thus only a brief overview is provided here. Reflective markers (14 $\mathrm{mm}$ spheres) were placed on the following body landmarks; mid-clavicle, 7th cervical vertebrae, right and left; shoulder, iliac crest, anterior superior iliac spine, posterior superior iliac spine, greater trochanter, medial epicondyle, lateral epicondyle, lateral malleouli, medial malleouli, heel, 5th, 2nd and 1st metatarsal heads using double-sided adhesive tape. Participants wore 'cluster sets' (4 reflective markers attached to a light weight rigid plastic shell) attached using Velcro elasticated wraps on the right and left thigh and shin to approximate the motion of these segments during dynamic trials. The pelvis and trunk cluster sets were attached using an elasticated belt and compression top, respectively. Three dimensional motions of these markers were collected whilst performing the pivoting using 10 Qualisys 'Oqus 7' (Model no. MCU 240) infrared cameras $(240 \mathrm{~Hz})$ operating through Qualisys Track Manager software (version 2.14). Ground reaction forces were collected from two AMTI (Model no. 600,900) force platforms $(1200 \mathrm{~Hz})$ embedded into the indoor surface. The force platform arrangement allowed data to be collected for both the PEN and FC.

From a standing trial, a 6-degree-of-freedom model of the lower extremity and trunk was created for each participant, 
including trunk, pelvis, thigh, shank and foot using Visual3D software (C-Motion, version 3.90.21). This kinematic model was used to quantify the motion at the hip, knee and ankle joints using Cardan angle sequence (Grood and Suntay 1983). The local coordinate system was defined at the proximal joint centre for each segment. The static trial position was designated as the subject's neutral (anatomical zero) alignment, and subsequent kinematic measures were related back to this position. Lower limb joint moments were calculated using an inverse dynamics approach (Winter 2009) through Visual3D software and are defined as external moments. Segmental inertial characteristics were estimated for each participant (Dempster 1955). The model utilised a CODA pelvis orientation (Bell et al. 1989) to define the location of the hip joint centre. The knee and ankle joint centres were defined as the mid-point of the line between lateral and medial markers. The trials were time normalised for each subject, with respect to the ground contact time of the COD task. Initial contact was defined as the instant after ground contact that the vertical GRF was higher than $20 \mathrm{~N}$ and end of contact was defined as the point where the vertical GRF subsided past $20 \mathrm{~N}$ for both PEN and FC. The weight acceptance phase of ground contact was defined as from the instant of initial contact to the point of maximum knee flexion during ground contact as used previously (Havens and Sigward 2015a; Jones et al. 2016a). Joint coordinate and force data were smoothed in Visual3D with a Butterworth low pass digital filter with cut-off frequencies of 12 and $25 \mathrm{~Hz}$, respectively. Cut off frequencies were selected based on a residual analysis (Winter 2009) and visual inspection of the data.

For comparisons between PEN and FC, peak vertical (Fz) and Horizontal (Fy) GRFs were determined along with peak hip, knee and ankle dorsi-flexion angles and peak hip, knee and ankle moments in the sagittal plane during weight acceptance, and analysed in Microsoft Excel (version 2016, Microsoft Corp., Redmond, WA, USA). Given whole body deceleration takes place in the sagittal plane during $180^{\circ} \mathrm{COD}$; therefore, only sagittal plane joint angles and moments were considered here. Furthermore, peak knee abduction angles and moments were calculated during the FC. Joint moment data were normalised to body mass $\left(\mathrm{Nm} \cdot \mathrm{kg}^{-1}\right)$. To evaluate deceleration strategy from PEN to FC, a FC/PEN contact horizontal (Fy component) GRF ratio (HGRFR) was also calculated (Jones et al. 2016a, 2016b).

\section{Statistical analysis}

All data are presented as mean $\pm S D$. The magnitude of differences between dependent variables were assessed as standardized mean difference (Cohens' $d$ effect sizes) and interpreted as trivial (0.0), small (0.2), moderate (0.6), and large (1.2), very large (2.0) and extremely large (4.0) (Hopkins 2002). Magnitude based inferences were used to determine whether differences were greater, trivial, or lower and classified as possibly (25.0-74.9\%), likely (75.0-94.9\%), very likely (95.9-99.5\%) and most likely (>99.5\%) (Hopkins et al. 2009). The effect was deemed unclear when the confidence interval spanned both substantial positive and substantial negative values $( \pm 0.2 \times$ between subject $S D)$. All statistical analyses were completed using Microsoft Excel (version 2016, Microsoft Corp., Redmond, WA, USA).

\section{Results}

Intraclass correlation coefficient (ICC) and coefficient of variation (CV) for COD trials were as follows: left limb (ICC $=0.91$; $2.5 \%$ ) and right limb (ICC $=0.83 ; \mathrm{CV}=2.9 \%)$.

Table 1 shows there was a possible small increase in time to completion while turning off the non-preferred limb $(3.00 \pm 0.19 \mathrm{~s})$ as compared to the preferred limb $(2.95 \pm 0.19 \mathrm{~s})$, with small ES $(0.23 ;+1.5 \%)$. Knee abduction angles in the non-preferred limb were very likely greater than those in the preferred limb $(E S=0.61 ;+74 \%)$ but this effect was only moderate. Knee abduction moments in the non-preferred limb were possibly greater than those in the preferred limb $(E S=0.28 ;+12 \%)$ but this effect was only small. Average HGRFR for the non-preferred limb was possibly greater than in the preferred limb $(E S=0.39 ;+7.5 \%)$, while differences in peak HGRFR between limbs was unclear.

Table 2 demonstrates average vertical GRF in the FC were most likely greater than those in the PEN during trials on both the preferred $(E S=3.01 ;+66.7 \%)$ and non-preferred limbs $(\mathrm{ES}=2.55 ;+77.6 \%)$. Peak vertical GRF in the FC were smaller than those in the PEN on both the preferred (ES $=1.61 ;-19 \%)$ and non-preferred limbs ( $E S=0.87 ;-21.4 \%$ ). Average horizontal GRF in the FC were most likely greater than those in the PEN during trials on both the preferred (ES $=3.67 ;+102.8 \%$ ) and non-preferred limbs ( $E S=3.05 ;+118.3 \%$ ). Differences in peak horizontal GRF between foot contacts for both preferred and non-preferred limbs were unclear.

Peak hip flexion angle in the FC were most likely smaller than those in the PEN on both the preferred (ES $=2.85 ;-30.1 \%$ ) and non-preferred limbs ( $E S=3.81 ;-25 \%)$. Peak knee flexion angle in the FC were most likely smaller than those in the PEN on both the preferred $(E S=5.74 ;-39.6 \%$ ) and non-preferred limbs $(E S=5.78 ;-39.1 \%)$. Peak ankle flexion angle in the FC were possibly smaller than those in the PEN for preferred limb

Table 1. Summary of braking strategy, knee joint mechanics and performance data during pivoting and inferences for difference of the means.

\begin{tabular}{|c|c|c|c|c|c|c|}
\hline Variable & Preferred & Non-Preferred & $\%$ diff & ES & $\begin{array}{c}\text { Chances } \\
\text { +ive/trivial/-ive }\end{array}$ & Inference \\
\hline Average HGRFR & $2.06 \pm 0.34$ & $2.26 \pm 0.72$ & $7.5 \pm 9.9$ & $0.39 \pm 0.50$ & $75 / 23 / 3$ & Possibly small $\uparrow$ \\
\hline Peak HGRFR & $0.99 \pm 0.22$ & $1.03 \pm 0.42$ & $1.2 \pm 16.4$ & $0.05 \pm 0.72$ & $36 / 36 / 27$ & Unclear \\
\hline Knee Abduction Angle $\left(^{\circ}\right)$ & $7.68 \pm 4.37$ & $11.17 \pm 3.65$ & $74.3 \pm 51.4$ & $0.61 \pm 0.32$ & $98 / 2 / 0$ & Very likely moderate $\uparrow$ \\
\hline Knee Abduction Moment $\left(\mathrm{Nm} \cdot \mathrm{kg}^{-1}\right)$ & $0.77 \pm 0.30$ & $0.85 \pm 0.31$ & $12.0 \pm 18.0$ & $0.28 \pm 0.39$ & $63 / 34 / 2$ & Possibly small $\uparrow$ \\
\hline Completion Time (s) & $2.95 \pm 0.19$ & $3.00 \pm 0.19$ & $1.5 \pm 2.1$ & $0.23 \pm 0.32$ & $57 / 41 / 2$ & Possibly small $\uparrow$ \\
\hline
\end{tabular}

Data displayed as mean \pm SD.

Trivial, small, moderate, large, and very large inference: possibly, 25-74.9\%; likely, 75-94.9\%; very likely, 95-99.5\%; and most likely, 95-99.5\%.

HGRFR = horizontal ground reaction force ratio. 


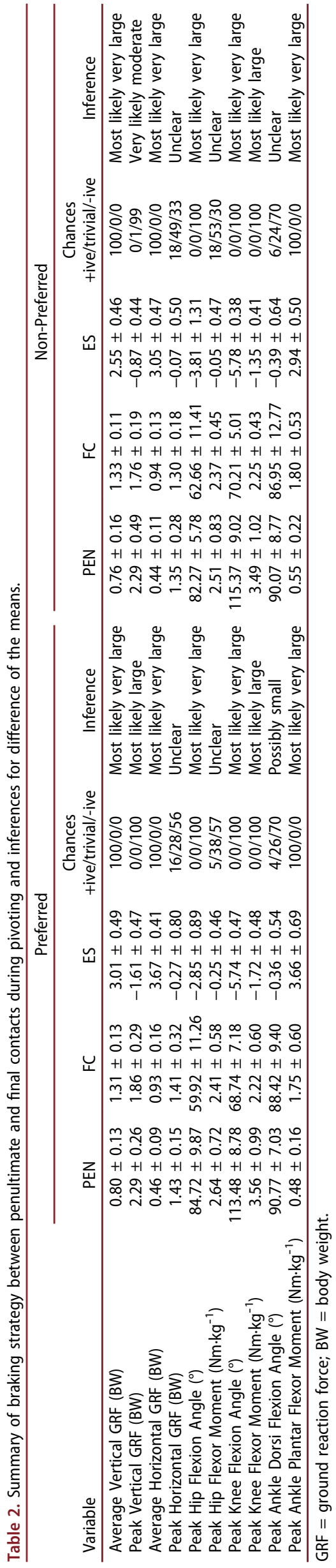

$(E S=0.36 ;-2.9 \%)$, while differences between foot contacts for the non-preferred limb were unclear.

Peak knee extensor moment in the FC were most likely smaller than those in the PEN on both the preferred $(E S=1.72 ;-37.8 \%)$ and non-preferred limbs $(E S=1.35$; $-33.9 \%)$. Peak ankle extensor moment in the FC were most likely greater than those in the PEN on both the preferred $(E S=3.66 ;+259 \%)$ and non-preferred limbs $(E S=2.94 ;+$ 235.6\%). All other kinetic differences between foot contacts were unclear.

\section{Discussion}

Although previous studies have considered the influence of the limb preference on knee injury risk factors during preplanned COD tasks (Brown et al. 2014, 2016; Greska et al. 2016), this is the first study to evaluate the interaction of PEN and FC on such factors. The aim of this study was to explore whether limb preference influences braking strategy during a $180^{\circ}$ pivot task in female soccer players. In addition, the study aimed to investigate differences in lower-limb kinematics and kinetics exist between preferred and non-preferred limbs. Consistent with previous work examining female athletes (Brown et al. 2014), the results of the current study indicate differences in knee joint mechanics between preferred and non-preferred limbs during a pre-planned pivot task. Furthermore, the results are in accord with recent work (Jones et al. 2016a) indicating that the PEN involved greater lower-limb joint flexion, peak vertical GRFs, but lower average vertical and horizontal GRFs compared to the FC. The current findings support our first hypothesis, that participants would display increased knee abduction angles when turning on the non-preferred limb compared to the preferred limb.

Previous work has demonstrated no differences in knee abduction angles between preferred and non-preferred limbs during $45^{\circ}$ cuts in female soccer players (Brown et al. 2014). In contrast, the current study found that very likely greater knee abduction angles existed when turning off non-preferred limb compared to the preferred limb. From our findings, there was a practically moderate $(E S=0.61)$ difference in knee abduction angles between limbs, however more studies on the current topic are therefore recommended. These findings are likely due to the greater associative knee joint loading during sharper changes of direction (Hader et al. 2014; Sigward et al. 2015; Havens and Sigward 2015a, 2015b). This finding, while preliminary, suggests that when turning off their nonpreferred limb, female soccer players may be at an increased risk of being exposed to greater knee abduction angles during the FC. Indeed, previous work has found knee abduction angle at initial contact to be a significant predictor of peak knee abduction moments during pivoting (Jones et al. 2015). The current study found possibly greater knee abduction moments in the non-preferred limb compared to the preferred limb, with small effects $(E S=0.28)$. This observation may support the hypothesis that an abducted knee position may create a GRF acting laterally outside the knee, thus increasing the moment arm between the knee joint axis and vertical GRF vector, leading to greater knee abduction moments. This finding has important implications for developing technique 
interventions during COD and agility training methods considering limb preference has been suggested to play a sexbased role in non-contact anterior cruciate ligament injury, specifically in soccer players (Brophy et al. 2010). Furthermore, increased knee abduction moments have been shown to increase ACL strain (Shin et al. 2009) and prospectively predict non-contact $A C L$ injury in female adolescent athletes (Hewett et al. 2005). Yet, this conflict in technique can be potentially facilitated by an athlete's physical capacity (i.e. ability to rapidly produce force and neuromuscular control) such that stronger athletes with optimal mechanics (i.e. no knee valgus) can tolerate the higher loads experiences and could, thus, adopt such techniques (Dos'Santos et al. 2018).

The current study found that with both limbs, increased peak vertical GRFs during the PEN relative to the $F C$ were observed; substantiating previous research on pivoting (Graham-Smith et al. 2009; Jones et al. 2016a). Another important finding was that average vertical and horizontal GRFs were higher in the FC. This result may be explained by the fact that during pivoting there is a need to reduce the body's horizontal velocity to zero before a reversal of direction, thus more substantial braking takes place during the FC. A note of caution is due here because of the absence of ground contact times, yet previous work (Jones et al. 2016a) has demonstrated greater horizontal braking impulse during the $F C$, due to longer ground contact times. It is therefore likely that such connections exist between GRF absorption through a greater range of motion, resulting in longer ground contact times and increased braking during the FC. These findings may help us to understand the braking demands of the FC in addition to knee joint position (knee abduction angle) and the potential for risk of injury. However, as previously mentioned certain body postures such as lateral trunk flexion (Dempsey et al. 2007), knee valgus (Kristianslund et al. 2014), limited knee flexion (Koga et al. 2010), lateral foot plant (Dempsey et al. 2007), and high ground reaction forces (Sigward et al. 2015) may induce greater loading but may be necessary for performance. Specifically, faster and sharper changes of direction increase knee joint loading but are also required for evading and escaping opponents in defensive and offensive situations, thus creating a 'performance-injury conflict'. Therefore, practitioners should pay close attention to the implications of changes in angle and/or velocity when designing and prescribing COD training to condition athletes to tolerate the physical demands of directional changes.

The joint moment data demonstrated similar findings to that for GRFs in that greater peak knee flexor moments were observed during PEN compared to final, in agreement with previous research (Graham-Smith et al. 2009). In contrast to earlier research, differences in peak hip extensor moment between limbs was unclear. The current study found that both limbs revealed greater ankle dorsi flexor moments during FC compared to PEN. These findings are in agreement with those obtained by (Jones et al. 2016a), who found greater ankle dorsi flexor moments during FC compared to PEN. These results may be explained by the fact that subjects initially made the FC with a forefoot plant, evoking an ankle dorsi flexor moment, whereas during PEN an initial rearfoot plant may have led to greater plantar flexor moments. These factors may explain the lack of between-limb differences for knee abduction moments, given previous research has shown rearfoot plants to produce greater knee abduction moments during $180^{\circ}$ pivoting (Cortes et al. 2012). Taken together, these findings indicate that the braking strategies when turning off both the preferred and non-preferred limbs in the sagittal plane has greater emphasis on counteracting knee flexor moments during the PEN, as compared with ankle dorsi flexor moments during the FC. Further research should be undertaken to investigate the role of hip flexor moments in the PEN and FC across both limbs, as previous research suggests joint loading is not consistent across joints (Havens and Sigward 2015b).

The present findings suggest female soccer players adopt similar lower-body joint postures regardless of whether trials were performed when turning off their preferred or nonpreferred limb. The joint angle data revealed that for both limbs, greater peak hip and knee flexion angles were observed during the PEN compared to FC. These results are consistent with previous research (Barber et al. 2015; Jones et al. 2016a; Thomas et al. 2017a, 2017b), that found peak hip and knee flexion angles to be significantly greater during the PEN compared to FC. These results are likely to be related to subjects adopting adjustments to body positions in the footfall prior to changing direction, which have been found to be advantageous for both performance (Graham-Smith et al. 2009; Dos' Santos et al. 2017; Jones et al. 2017) and reduced loading (Graham-Smith et al. 2009; Jones et al. 2016b). It is possible, therefore, that greater hip and knee flexion are required to absorb loading through a greater range of motion, facilitating longer braking force application, thus impulse, resulting in a greater reduction in velocity. The combined hip and knee flexion is maintained in the transition period from PEN to FC to allow optimal position in preparation for FC. These results are in accord with recent work indicating that the PEN plays a pivotal role in the interaction between strength (GrahamSmith et al. 2009; Jones et al. 2017), speed (Jones et al. 2017), and technique (Graham-Smith et al. 2009; Dos' Santos et al. 2017) during $180^{\circ}$ pivoting. Therefore, researchers and practitioners are encouraged to consider the role of the PEN when coaching and evaluating COD technique. It is possible, therefore, that the PEN plays a role in preparing the body for an optimal position for FC when turning off both preferred and non-preferred limbs. Specifically, a large centre of pressurecentre of mass distance (i.e. placement of the PEN in front of the body to create a backward lean of the trunk) will maximize horizontal braking force to reduce momentum (impulsemomentum relationship). This allows the knee (and hip) to go through a greater range of flexion to absorb loading through greater range of motion, facilitating longer braking force application, thus impulse, resulting in a greater reduction in velocity (Impulse $=$ change in momentum). Furthermore, hip and knee flexion is maintained from the PENFC to allow for optimal body position in preparation for FC whereby the trunk will remain upright or slightly forward and athletes may rotate their whole nobody during the flight phase between PEN and FC to effectively align themselves into the new intended direction.

A limitation of the current study is the pre-planned execution of the COD task, whereas unanticipated COD has been 
shown to elevate knee joint loads during cutting (Besier et al. 2001). Furthermore, the findings of the current study can only be extrapolated to female soccer participants performing $180^{\circ}$ pivoting. Another limitation of the current study was the relatively low sample size, thus future studies should examine the influence of limb preference in COD with greater sample sizes. Except for knee abduction angles and moments, this study only featured lower-limb joint angles and moments in the sagittal plane. Despite hip abduction and rotation angles, such as the motion on the frontal and transversal planes, are commonly investigated in cutting studies (Kristianslund and Krosshaug 2013; Kristianslund et al. 2014), whole body deceleration takes place in the sagittal plane during $180^{\circ}$ pivoting. In future studies, it might be possible to investigate the influence of these parameters on braking strategy and knee joint mechanics. Furthermore, several researchers (Welsh and Knight 2015; Sainani 2018) have criticized magnitude-based inference for its high type I error rates and incorrect definitions, and that confidence intervals or Bayesian analysis should be used as methods of statistical inference. However, Batterham and Hopkins have responded to these criticisms (Batterham and Hopkins 2015, 2019; Hopkins and Batterham 2018).

\section{Practical application}

The study has shown that female soccer players demonstrate similar braking strategies when turning off the preferred and non-preferred limbs. However, greater knee abduction angles and moments may exist when turning off the non-preferred limb compared to the preferred limb. According to these data, it seems that limb preference does not impact biomechanical characteristics of COD performance in female soccer players, yet knee abduction angles and moments should be monitored with caution. Notwithstanding, practitioners are encouraged to assess COD biomechanics on both limbs to identify potential differences in kinetic and kinematic differences, and global performance measures. As a result, individualised preventative training interventions can be created as part of an ongoing monitoring programme. Finally, it would be advantageous that athletes are equally proficient in changing direction effectively off both limbs due to the unpredictable nature of soccer.

\section{Disclosure statement}

No potential conflict of interest was reported by the authors.

\section{Funding}

No funding was received for this study from any organization.

\section{References}

Ade J, Fitzpatrick J, Bradley PS. 2016. High-intensity efforts in elite soccer matches and associated movement patterns, technical skills and tactical actions. Information for position-specific training drills. J Sports Sci. 34:2205-2214.

Barber OR, Thomas C, Jones PA, McMahon JJ, Comfort P. 2015. Reliability of the 505 change of direction test in netball players. Int J Sports Physiol Perform. 11(3):377-380
Batterham A, Hopkins W. 2019. The problems with "The problem with 'Magnitude-Based Inference'". Medicine \& Science in Sports \& Exercise; p. 51.

Batterham AM, Hopkins WG. 2015. The case for magnitude-based inference. Med Sci Sports Exerc. 47:885.

Bell AL, Brand RA, Pedersen DR. 1989. Prediction of hip joint centre location from external landmarks. Hum Mov Sci. 8:3-16.

Besier TF, Lloyd DG, Ackland TR, Cochrane JL. 2001. Anticipatory effects on knee joint loading during running and cutting maneuvers. Med Sci Sports Exerc. 33:1176-1181.

Bloomfield J, Polman R, O'Donoghue P. 2007. Turning movements performed during FA Premier League soccer matches. J Sports Sci Med, 6: 9

Brophy R, Silvers HJ, Gonzales T, Mandelbaum BR. 2010. Gender influences: the role of leg dominance in $A C L$ injury among soccer players. $\mathrm{Br}$ J Sports Med. 44:694-697.

Brown N, Donelon T, Smith LC, Jones PA. 2016. An investigation into the effect of limb preference on knee mechanics and braking strategy during a $180^{\circ}$ change of direction task: an exploratory study. J Sports Sci. 34:s57. doi:10.1080/02640414.2016.1260807

Brown SR, Wang H, Dickin DC, Weiss KJ. 2014. The relationship between leg preference and knee mechanics during sidestepping in collegiate female footballers. Sports Biomech. 13:351-361.

Cortes N, Morrison S, Van Lunen BL, Onate JA. 2012. Landing technique affects knee loading and position during athletic tasks. J Sci Med Sport. 15:175-181.

Dempsey AR, Lloyd DG, Elliott BC, Steele JR, Munro BJ. 2009. Changing sidestep cutting technique reduces knee valgus loading. Am J Sports Med. 37:2194-2200.

Dempsey AR, Lloyd DG, Elliott BC, Steele JR, Munro BJ, Russo KA. 2007. The effect of technique change on knee loads during sidestep cutting. Med Sci Sports Exerc. 39:1765-1773.

Dempster WT. 1955. Space requirements of the seated operator: geometrical, kinematic, and mechanical aspects of the body, with special reference to the limbs. Michigan State Univ East Lansing.

Dos' Santos T, Thomas C, Jones PA, Comfort P. 2017. Mechanical determinants of faster change of direction speed performance in male athletes. J Strength Cond Res. 31:696-705.

Dos'Santos T, Thomas C, Comfort P, Jones PA. 2018. The effect of angle and velocity on change of direction biomechanics: an angle-velocity trade-off. Sports Med. 48:2235-2253.

Dos'Santos T, Thomas C, Comfort P, Jones PA. 2019. Role of the penultimate foot contact during change of direction: implications on performance and risk of injury. Strength Cond J, 41(1):87-104.

Graham-Smith P, Atkinson L, Barlow R, Jones P, 2009. Braking characteristics and load distribution in 180 degree turns. Presented at the The Proceedings of the 5th annual UKSCA conference, p. 6-7.Wyboston Lakes, United Kingdom.

Greig M. 2009. The influence of soccer-specific activity on the kinematics of an agility sprint. Euro J Sport Sci. 9:23-33. doi:10.1080/17461390802579129

Greska EK, Cortes N, Ringleb SI, Onate JA, Van Lunen BL. 2017. Biomechanical differences related to leg dominance were not found during a cutting task. Scand J Med Sci Sports. 27(11): 1328-1336.

Grood ES, Suntay WJ. 1983. A joint coordinate system for the clinical description of three-dimensional motions: application to the knee. J Biomech Eng. 105:136-144.

Hader K, Mendez-Villanueva A, Ahmaidi S, Williams BK, Buchheit M. 2014. Changes of direction during high-intensity intermittent runs: neuromuscular and metabolic responses. BMC Sports Sci Med Rehabil. 6:2

Havens KL, Sigward SM. 2015a. Whole body mechanics differ among running and cutting maneuvers in skilled athletes. Gait Posture. 42:240-245. doi:10.1016/j.gaitpost.2014.07.022

Havens KL, Sigward SM. 2015b. Joint and segmental mechanics differ between cutting maneuvers in skilled athletes. Gait Posture. 41:33-38. doi:10.1016/j.gaitpost.2014.08.005

Hewett TE, Myer GD, Ford KR, Heidt RS, Colosimo AJ, McLean SG, Van Den Bogert AJ, Paterno MV, Succop P. 2005. Biomechanical measures of neuromuscular control and valgus loading of the knee predict anterior cruciate ligament injury risk in female athletes a prospective study. Am J Sports Med. 33:492-501. 
Hopkins W, Marshall S, Batterham A, Hanin J. 2009. Progressive statistics for studies in sports medicine and exercise science. Med Sci Sports Exerc. 41:3.

Hopkins WG, 2002. A scale of magnitudes for effect statistics. A new view of statistics http://sportsci.org/resource/stats/effectmag.html.

Hopkins WG, Batterham AM. 2018. The vindication of magnitude-based inference. Sportsci. 22:19-29.

Jones P, Bampouras TM, Marrin K. 2009. An investigation into the physical determinants of change of direction speed. J Sports Med Phys Fitness. 49:97-104.

Jones PA, Herrington L, Graham-Smith P. 2016a. Braking characteristics during cutting and pivoting in female soccer players. J Electromyogr Kinesiol. 30:46-54.

Jones PA, Herrington LC, Graham-Smith P. 2015. Technique determinants of knee joint loads during cutting in female soccer players. Hum Mov Sci. 42:203-211. doi:10.1016/j.humov.2015.05.004

Jones PA, Herrington LC, Graham-Smith P. 2016b. Technique determinants of knee abduction moments during pivoting in female soccer players. Clin Biomech. 31:107-112.

Jones PA, Thomas C, Dos'Santos T, McMahon JJ, Graham-Smith P. 2017. The role of eccentric strength in $180^{\circ}$ turns in female soccer players. Sports. 5:42.

Koga H, Nakamae A, Shima Y, Iwasa J, Myklebust G, Engebretsen L, Bahr R, Krosshaug T. 2010. Mechanisms for noncontact anterior cruciate ligament injuries: knee joint kinematics in 10 injury situations from female team handball and basketball. Am J Sports Med. 38:2218-2225.

Kristianslund E, Faul O, Bahr R, Myklebust G, Krosshaug T. 2014. Sidestep cutting technique and knee abduction loading: implications for $\mathrm{ACL}$ prevention exercises. Br J Sports Med. 48:779-783.
Kristianslund E, Krosshaug T. 2013. Comparison of drop jumps and sport-specific sidestep cutting: implications for anterior cruciate ligament injury risk screening. Am J Sports Med, 41.

Robinson G, O'Donoghue P, Wooster B. 2011. Path changes in the movement of English Premier League soccer players. J Sports Med Phys Fitness. 51:220-226.

Sainani KL. 2018. The problem with" Magnitude-based Inference". Med Sci Sports Exerc. 50:2166-2176.

Shin CS, Chaudhari AM, Andriacchi TP. 2009. The effect of isolated valgus moments on $A C L$ strain during single-leg landing: a simulation study. J Biomech. 42:280-285.

Sigward SM, Cesar GM, Havens KL. 2015. Predictors of frontal plane knee moments during side-step cutting to 45 and 110 degrees in men and women: implications for anterior cruciate ligament injury. Clin J Sport Med. 25:529-534. doi:10.1097/jsm.0000000000000155

Thomas C, Dos'Santos T, Kyriakidou I, Cuthbert M, Fields C, Jones PA, 2017a. A comparison of braking characteristics between modified and traditional 505 change of direction tasks in female netball players: an exploratory study.

Thomas C, Dos'Santos T, Kyriakidou I, Cuthbert M, Fields C, Jones PA, 2017b. An investigation into the effect of limb preference on knee mechanics and braking strategy during pivoting in female soccer players: an exploratory study.

Welsh AH, Knight EJ. 2015. "Magnitude-based inference": a statistical review. Med Sci Sports Exerc. 47:874.

Winter DA. 2009. Biomechanics and motor control of human movement. John Wiley \& Sons.

Young WB, Henry B, Dawson G. 2015. Agility and change-of-direction speed are independent skills: implications for training for agility in invasion sports. Int J Sports Sci Coach. 10:159-169. 